\title{
Flagellin delays spontaneous human neutrophil apoptosis
}

\author{
Gabriela V Salamone ${ }^{1,2}$, Yanina Petracca ${ }^{1}$, Juan I Fuxman Bass ${ }^{1,3}$, Martín Rumbo ${ }^{4}$, Karen A Nahmod ${ }^{1}$, \\ Maria L Gabelloni ${ }^{1,3}$, Mónica E Vermeulen ${ }^{1,2}$, Mario J Matteo ${ }^{2}$, Jorge R Geffner ${ }^{1,2}$ and Analia S Trevani ${ }^{1,2}$
}

Neutrophils are short-lived cells that rapidly undergo apoptosis. However, their survival can be regulated by signals from the environment. Flagellin, the primary component of the bacterial flagella, is known to induce neutrophil activation. In this study we examined the ability of flagellin to modulate neutrophil apoptosis. Neutrophils cultured for 12 and $24 \mathrm{~h}$ in the presence of flagellin from Salmonella thyphimurim at concentrations found in pathological situations underwent a marked prevention of apoptosis. In contrast, Helicobacter pylori flagellin did not affect neutrophil survival, suggesting that Salmonella flagellin exerts the antiapoptotic effect by interacting with TLR5. The delaying in apoptosis mediated by Salmonella flagellin was coupled to higher expression levels of the antiapoptotic protein Mcl-1 and lower levels of activated caspase-3. Analysis of the signaling pathways indicated that Salmonella flagellin induced the activation of the p38 and ERK1/2 MAPK pathways as well as the PI3K/Akt pathway. Furthermore, it also stimulated $I \kappa B \alpha$ degradation and the phosphorylation of the p65 subunit, suggesting that Salmonella flagellin also triggers NF- $\kappa$ B activation. Moreover, the pharmacological inhibition of ERK1/2 pathway and NF- $\kappa$ B activation partially prevented the antiapoptotic effects exerted by flagellin. Finally, the apoptotic delaying effect exerted by flagellin was also evidenced when neutrophils were cultured with whole heat-killed S. thyphimurim. Both a wild-type and an aflagellate mutant S. thyphimurim strain promoted neutrophil survival; however, when cultured in low bacteria/neutrophil ratios, the flagellate bacteria showed a higher capacity to inhibit neutrophil apoptosis, although both strains showed a similar ability to induce neutrophil activation. Taken together, our results indicate that flagellin delays neutrophil apoptosis by a mechanism partially dependent on the activation of ERK1/2 MAPK and NF- $\kappa$ B. The ability of flagellin to delay neutrophil apoptosis could contribute to perpetuate the inflammation during infections with flagellated bacteria.

Laboratory Investigation (2010) 90, 1049-1059; doi:10.1038/labinvest.2010.77; published online 5 April 2010

KEYWORDS: flagellin; neutrophil; apoptosis

Neutrophils are short-lived professional phagocytes that are generally regarded as terminally differentiated cells. They are the most abundant type of immune cell in the circulation, being the first line of defense against bacterial and fungal infections. Neutrophils are rapidly and massively recruited from the circulation into inflammatory sites. ${ }^{1-2}$ Although they are key antiinfectious actors, they are also inflammatory cells that can mediate tissue damage. Thus, neutrophil lifespan must be strongly regulated because, although extended survival is beneficial for pathogen elimination, apoptosis is necessary to prevent the release of their cytotoxic content to the inflammatory site. ${ }^{3}$
A specific feature of neutrophils is their ability to rapidly undergo spontaneous apoptosis. ${ }^{3}$ However, at inflammatory foci, the expression of their apoptotic program can be regulated by a number of agents such as cytokines, pathogens, and environmental stressors. It has been described that granulocyte-macrophage colony-stimulating factor (GM-CSF), interleukin (IL)-1, IL-2, leukotriene B4, C5a, lipopolysaccharide (LPS), and corticosteroids inhibit neutrophil apoptosis, ${ }^{4-6}$ whereas proteolytic enzymes, immune complexes, bacteria, and viruses stimulate neutrophil apoptosis. ${ }^{7-9}$

Many clinically relevant pathogens are flagellate, including agents responsible for gastroenteritis (Salmonella, diarrheagenic

\footnotetext{
'Departamento de Inmunología, Instituto de Investigaciones Hematológicas and Instituto de Estudios Oncológicos 'Fundación Maissa', Academia Nacional de Medicina, Buenos Aires, Argentina; ${ }^{2}$ Departamento de Microbiología, Facultad de Medicina, Universidad de Buenos Aires, Buenos Aires, Argentina; ${ }^{3}$ Departamento de Fisiología y Biología Molecular y Celular, Facultad de Ciencias Exactas y Naturales, Universidad de Buenos Aires, Buenos Aires, Argentina and ${ }^{4}$ Laboratorio de Investigaciones en el Sistema Inmune, Facultad de Ciencias Exactas, UNLP, La Plata, Argentina Correspondence: Dr GV Salamone, PhD, or Dr AS Trevani, PhD, IIHEMA-Academia Nacional de Medicina, Pacheco de Melo 3081, 1425 Buenos Aires, Argentina. E-mail: salamone.gabriela@gmail.com or analiatrevani@yahoo.com.ar 
Escherichia coli, Campylobacter), pneumonia (Pseudomonas, Burkholderia, Legionella), and invasive infections (Proteus, E. coli, Clostridium, etc). Flagella are whip-like organelles that attach to a rotary motor embedded in the bacterial cell wall, providing motility to the organism. The body of the flagellum consists of a mass of protofilaments, each of which is a long, end-to-end polymer of a single protein, flagellin. ${ }^{10}$ Because of the physical constraints imposed by its function, flagellin has a relatively conserved structure even among widely diverse bacterial species. ${ }^{10}$ Flagella are essential structures for the pathogenic potential of bacteria because they provide motility, a property that in some cases is absolutely necessary for the bacterium to colonize its mammalian hosts, and in other cases, provide an advantage in reaching particular niches in one or more of its reservoirs or hosts that it colonizes. ${ }^{11}$ The bacterial expression of flagella is highly regulated, likely because of both its high metabolic cost and because flagella/ flagellin is targeted by the mammalian immune system to detect the presence of motile organisms. ${ }^{11}$ In fact, flagellin shows microbial-associated molecular pattern features, including sequence conservation and wide distribution among bacteria. Flagellin recognition by the cell surface receptor Toll-like receptor 5 (TLR5) triggers the activation of different cell types, promoting gene expression designed to protect the host from perturbing microbes. ${ }^{12-13}$ Although TLR5 is the only identified surface receptor for flagellin, an intracellular receptor has also been recognized. Indeed, a member of the NOD-like receptor family, the NLRC4, also known as Ipaf, has been shown to trigger caspase- 1 and IL- $1 \beta$ secretion in response to cytoplasmic flagellin in a TLR5-independent manner. ${ }^{14}$ To exert this effect, flagellin is required to be translocated to the cytoplasm, apparently by using a type 3 or type 4 secretion system.

By stimulating the TLR5, flagellin induces responses in epithelial cells, endothelial cells, macrophages, dendritic cells, and $\mathrm{T}$ cells. ${ }^{10,15-17}$ In addition, flagellin activates TLR5 on natural $\mathrm{CD}^{+} / \mathrm{CD} 25^{+}$T-regulatory cells, leading to increased suppressive activity, suggesting that flagellin has a complex role in bridging innate immunity and adaptive immunity. ${ }^{18}$ Previous studies also showed the expression of both TLR5 mRNA and protein in human neutrophils and the ability of flagellin to stimulate different neutrophil responses such as the cell shape change, the production of the chemokine IL-8, the shedding of L-selectin, and a priming effect on superoxide anion production triggered by FMLP. ${ }^{19}$ Differences in the course of the infection by wild-type and aflagellate Salmonella have been previously described. ${ }^{20}$ These differences could be related not only with the ability of flagellin to stimulate a number of inflammatory responses mediated by neutrophils, but also with the potential role of flagellin in the control of neutrophil survival. In this regard, premature neutrophil apoptosis might result in failure of host defense, whereas a delay in their apoptosis might provide sufficient time for the host to generate defensive responses and/or to induce tissue injury. Thus, this study was conducted to evaluate the effect of flagellin on neutrophil survival.

\section{MATERIALS AND METHODS}

The studies performed in this work have been reviewed and approved by the Academia Nacional de Medicina Review Board and Ethical Committee. All blood donors provided written informed consent for the collection of samples and subsequent analysis.

\section{Abs and Reagents}

Endotoxin-free reagents and plastics were used in all experiments. RPMI-1640 and PBS were purchased from HyClone Laboratories (Logan, UT, USA). Fetal calf serum (FCS) and penicillin/streptomycin were purchased from Invitrogen Life Technologies (Grand Island, NY, USA). Fluorescein isothiocyanate (FITC)-conjugated anti-human CD62L was purchased from Immunotech (Marseille, France). Rabbit polyclonal Abs against Thr180/Tyr182-phosphorylated p38 MAPK (catalog no. 9211), Thr308-phosphorylated Akt (catalog no. 9275), mouse mAb against Thr202/Tyr204phosphorylated ERK1/ERK2 (catalog no. 9106), mouse mAb against caspase-3 (catalog no. 9668), and HRP-conjugated goat anti-mouse $\mathrm{Ab}$ (catalog no. 7076) were obtained from Cell Signalling Technology (Danvers, MA, USA). Anti-p65 (catalog no. sc-372), anti- $\mathrm{I} \kappa \mathrm{B}$ rabbit polyclonal Ab (catalog no. 554135), anti-Mcl-1 mAb (catalog no. 559027), anti-active caspase-3 mAb (catalog no. 559341), and HRP-conjugated goat anti-rabbit IgG Ab (catalog no. 554021) were purchased from BD Pharmingen (Franklin Lakes, NJ, USA). Anti-FAS mAb (catalog no. PN IM1504) was from Beckman Coulter (Brea, CA, USA). PD98059 (MEK inhibitor), SB203580 (p38 MAPK inhibitor), and wortmannin were obtained from Calbiochem (Gibbstown, NJ, USA); and Bay 11-7082, acridine orange, and ethidium bromide were purchased from Sigma-Aldrich (St Louis, MO, USA). The synthetic lipopeptide Pam3-Cys-Ser-Lys4 (Pam3CSK4) was purchased from InvivoGen Corporation (Rockville, MD, USA).

\section{Bacterial Strains and Flagellin Purification}

Salmonella enterica serovar typhimurium ATCC14028, S. typhimurium SIN22 strain (flagellate) and its isogenic mutant SIN41 strain (aflagellate), as well as Bacillus cereus B14128 strain (local isolated strain, Cátedra de Microbiología General, FCE, UNLP), were all grown in LB media for $16 \mathrm{~h}$ at $37^{\circ} \mathrm{C}$ with mild agitation (80 r.p.m.) to prevent flagellum release. Helicobacter pylori locally isolated strains were cultured as previously described. ${ }^{21}$ Flagellins from S. enterica serovar typhimurium ATCC 14028 (FliC), from B. cereus, and from $H$. pylori were purified as previously described. ${ }^{17}$ Bacteria were harvested by centrifugation (5000 r.p.m. for $30 \mathrm{~min}$ ), washed once with PBS and then suspended at a high concentration. Flagella were sheared from surface by extensive vortexing, and pelleted by ultracentrifugation (30000 r.p.m. for 2 h). Resuspended flagella were then heated 


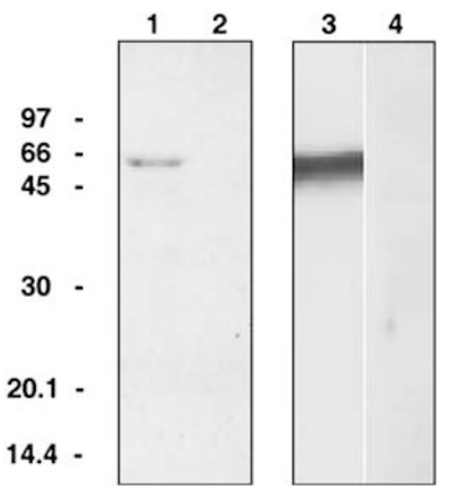

Figure 1 Analysis of the purity of FliC preparations. Untreated (lanes 1 and 3) or trypsinized flagellin preparations (lanes 2 and 4) were subjected to SDS-PAGE (10\% gels) and visualized by staining with Coomassie brilliant blue (lanes 1 and 2) or were electrotransferred and immunoblotted with a specific mAb (2B3H10a; lanes 3 and 4$)$.

to release flagellin monomers and ultracentrifuged again in the same condition, keeping the supernatant with the soluble flagellin. Subsequently, flagellin was depleted of endotoxin activity by using Detoxi-Gel Affinity Columns (Pierce). The samples were then concentrated in PBS and stored at $-80^{\circ} \mathrm{C}$. Using the Limulus Amebocyte Lysate Pyrotell (Associates of Cape Cod), the residual LPS concentration was determined to be $<20 \mathrm{pg} / \mu \mathrm{g}$ FliC. Where indicated, flagellins were treated for $2 \mathrm{~h}$ at $37^{\circ} \mathrm{C}$ with $0.25 \%$ trypsin-EDTA (Invitrogen, Carlsbad, CA, USA) to totally hydrolyze the proteins, followed by heating at $70^{\circ} \mathrm{C}$ for $1 \mathrm{~h}$ to inactivate the trypsin. Purity of the flagellin preparations and the efficiency of trypsin digestion were checked using Coomassie-stained protein gels and standard SDS-PAGE and immunoblotting with $\mathrm{mAb}$ 2B3H10a, a FliC-specific monoclonal Ab developed at Dr Rumbo's laboratory (Figure 1).

\section{Blood Samples}

Blood samples were obtained from healthy donors who had taken no medication for at least 10 days before the day of sampling. Blood was obtained by venipuncture of the forearm vein, and it was drawn directly into heparinized plastic tubes.

\section{Neutrophil Isolation}

Neutrophils were isolated by Ficoll-Hypaque gradient centrifugation and dextran sedimentation (Ficoll and dextran, GE Healthcare, Munich, Germany; Hypaque, Winthrop Products, Buenos Aires, Argentina), as previously described. ${ }^{22}$ Cells, suspended in RPMI-1640 supplemented with $1 \%$ heat-inactivated FCS (complete medium), contained $>96 \%$ neutrophils, as determined by May-GrunwaldGiemsa-stained cytopreps. All neutrophil preparations were FACs analyzed after purification to guarantee that their FSC/ SSC parameters were compatible with those of nonactivated cells. Levels of monocyte contamination were always
$<0.25 \%$, as evaluated by CD14 staining and flow cytometry. To minimize neutrophil spontaneous activation, cells were used immediately after isolation.

\section{Cell Cultures}

Neutrophils $\left(100 \mu \mathrm{l}, 4 \times 10^{6} / \mathrm{ml}\right)$ were seeded into flatbottomed 96-well plates and, unless otherwise stated, they were cultured in the presence or absence of $10 \mathrm{ng} / \mathrm{ml}$ of heattreated FliC for $24 \mathrm{~h}$ at $37^{\circ} \mathrm{C}$ in a humidified atmosphere with $5 \% \mathrm{CO}_{2}$. Apoptosis was then evaluated either by fluorescent microscopy or flow cytometry.

\section{Quantitation of Cellular Apoptosis and Viability by Fluorescent Microscopy}

Quantitation was performed as previously described ${ }^{23}$ using the fluorescent DNA-binding dyes acridine orange $(100 \mu \mathrm{g} / \mathrm{ml})$ to determine the percentage of cells that had undergone apoptosis, and ethidium bromide $(100 \mu \mathrm{g} / \mathrm{ml})$ to differentiate between viable and nonviable cells. To assess the percentage of cells showing morphologic features of apoptosis, at least 200 cells were scored in each experiment. Previous observations have shown that morphological assessment of neutrophil apoptosis closely correlates with results obtained using other methods to assay apoptosis, such as propidium iodide staining and annexin- $\mathrm{V}$ binding. ${ }^{24}$

\section{Quantitation of Neutrophil Apoptosis by Annexin-V Binding and Flow Cytometry}

Annexin- $\mathrm{V}$ binding to neutrophils was performed using an apoptosis detection kit (Immunotech, Marseille, France). In brief, cells were labeled with annexin-V-FITC for $20 \mathrm{~min}$ at $4{ }^{\circ} \mathrm{C}$ and with propidium iodide immediately before evaluation of fluorescence by flow cytometry (FACScan flow cytometer; Becton Dickinson, San Jose, CA, USA). Collected data were analyzed by using the CellQuest analysis software (Becton Dickinson), as previously described. ${ }^{25}$ Results are reported as percentage of annexin-V-positive cells.

\section{Western Blotting}

Neutrophils $\left(4 \times 10^{6}\right)$, suspended in complete medium, were pre-warmed for $15 \mathrm{~min}$ at $37^{\circ} \mathrm{C}$ and were then stimulated with heat-treated FliC $50 \mathrm{ng} / \mathrm{ml}$ for $0-120 \mathrm{~min}$ at $37^{\circ} \mathrm{C}$. The reactions were terminated by addition of $1 \mathrm{ml}$ of ice-cold saline with $1 \mathrm{mM}$ PMSF and rapid centrifugation. The pellets were immediately frozen in dry ice after aspiration of the supernatants. They were then dissolved in sample buffer (2\% SDS, $10 \%$ glycerol, 5\% 2-mercaptoethanol, and trace amounts of bromophenol blue dye in $62.5 \mathrm{mM}$ Tris- $\mathrm{HCl}, \mathrm{pH}$ 6.8), heated for $5 \mathrm{~min}$ at $95^{\circ} \mathrm{C}$, and stored at $-70^{\circ} \mathrm{C}$ until subjected to gel electrophoresis. After SDS-PAGE, proteins were electotransferred from the gel to polyvinylidene difluoride (PVDF) membranes (Millipore Bedford, MA, USA) for $2 \mathrm{~h}$ and then blocked in PBS containing 0.05\% Tween-20 and $5 \%$ nonfat milk or $2.5 \%$ bovine serum albumin (BSA) for $1 \mathrm{~h}$ at $25^{\circ} \mathrm{C}$. The membranes were immunoblotted with 
the indicated antibodies overnight at $4{ }^{\circ} \mathrm{C}$. After washing, bound antibodies were visualized with HRP-conjugated antibodies against rabbit or mouse immunoglobulin G (IgG) by using the enhanced chemiluminesence (ECL) Western Blotting System (GE Healthcare, Freiburg, Germany).

\section{Analysis of the Expression of Intracellular Mcl-1 by Flow Cytometry}

Neutrophils $\left(1 \times 10^{6}\right)$ were cultured in the presence or absence of heat-treated FliC $(10 \mathrm{ng} / \mathrm{ml})$ for $24 \mathrm{~h}$ at $37^{\circ} \mathrm{C}$. They were then fixed with $2 \%$ formaldehyde for $15 \mathrm{~min}$ at $4{ }^{\circ} \mathrm{C}$, washed with saline and permeabilized with methanol for $5 \mathrm{~min}$ at $4{ }^{\circ} \mathrm{C}$. Cells were then suspended in saline supplemented with glycine $(0.1 \mathrm{mg} / \mathrm{ml})$, incubated with anti-Mcl-1 or matched isotype control antibodies, and the intracellular expression of Mcl-1 was analyzed by flow cytometry using the FITC-conjugated rabbit anti-mouse IgG.

\section{Analysis of Caspase-3 Activation by Flow Cytometry}

Neutrophils $\left(1 \times 10^{6}\right)$ were cultured in the presence or absence of heat-treated FliC $(10 \mathrm{ng} / \mathrm{ml})$ for $24 \mathrm{~h}$ at $37^{\circ} \mathrm{C}$. Cells were then fixed and permeabilized using IntraStain kit (Dako, Glostrup, Denmark) following the manufacturer's instructions. After labelling with the FITC-conjugated rabbit anti-active caspase-3 mAb (catalog no. 559341; BD Pharmingen) for $30 \mathrm{~min}$ at room temperature, fluorescence was evaluated by flow cytometry.

\section{Measurement of IL-8 Production}

Neutrophils suspended in complete medium $\left(2 \times 10^{6} / \mathrm{ml}\right)$ were seeded into round-bottomed 96-well plates and were cultured in the presence or absence of flagellate or aflagellate bacteria for $3 \mathrm{~h}$ at $37^{\circ} \mathrm{C}$. Culture supernatants were then collected, centrifuged, and IL-8 concentration was measured by ELISA (R\&D Systems).

\section{Neutrophil Shape Change}

Neutrophils $\left(2 \times 10^{6} / \mathrm{ml}\right)$ suspended in complete medium were incubated in a shaking water bath for $5 \mathrm{~min}$ at $37^{\circ} \mathrm{C}$ in the presence or absence of flagellate or aflagellate bacteria. Cells were then centrifuged, suspended in PBS, and fixed by the addition of an equal volume of glutaraldehyde (1\%) in PBS. Neutrophil shape change was evaluated by flow cytometry and results were expressed as the shift in the forward light scatter values in stimulated cells compared with mockstimulated neutrophils.

\section{Statistical Analysis}

Statistical significance was determined using the nonparametric Friedman's test for multiple comparisons with Dunn's posttest or Student's $t$-test. Statistical significance was defined as $P<0.05$.

\section{RESULTS \\ Flagellin Delays Neutrophil Apoptosis}

In a first set of experiments, we determined whether Salmonella flagellin was able to modulate neutrophil spontaneous apoptosis rate. Cells were cultured for $24 \mathrm{~h}$ in the absence or presence of different concentrations of FliC and the percentage of apoptotic cells was then evaluated by fluorescence microscopy. Figure $2 \mathrm{a}$ shows that heat-treated FliC, used at 1, 10, 50, 100, and $300 \mathrm{ng} / \mathrm{ml}$, significantly delayed neutrophil apoptosis. Similar results were observed when apoptosis was evaluated using FITC-annexin-V and flow cytometry (Figure 2b). It is noteworthy that unheated FliC used at $10 \mathrm{ng} / \mathrm{ml}$, a concentration at which greater antiapoptic effects of heat-treated FliC were observed, did not modulate neutrophil survival (Figure 2a). Although LPS concentrations in FliC preparation were always lower than $20 \mathrm{pg} / \mu \mathrm{g}$ FliC, we performed additional assays to rule out the possibility that the antiapoptotic effect mediated by flagellin could be because of the presence of contaminating LPS. We found that digestion of flagellin with trypsin completely abrogated its ability to delay neutrophil apoptosis (Figure $2 \mathrm{~b}$ ). Moreover, the addition of polimixin $\mathrm{B}$ to cell cultures did not modify the effects of FliC on neutrophil apoptosis (data not shown). These findings are in accordance with the fact that at concentrations of the FliC used in our assays $(10-300 \mathrm{ng} / \mathrm{ml}$ ), the maximal levels of LPS that could be present in the cultures were $<6 \mathrm{pg} / \mathrm{ml}$, a concentration at which LPS had no effect on neutrophil apoptosis (data not shown). The specificity of the response was further confirmed by the fact that flagellin from $H$. pylori, which is not recognized by TLR $5,{ }^{26}$ was unable to delay neutrophil apoptosis (Figure $2 \mathrm{c}$ and $\mathrm{d}$ ).

It is noteworthy that flagellin from $B$. cereus also significantly delayed neutrophil apoptosis, supporting the fact that the antiapoptotic effect of flagellin is not strain restricted (Figure 2e and f). Interestingly, the apoptosis-delaying effect of flagellin was also observed at earlier time points $(12 \mathrm{~h}$ ) when only a reduced proportion of the population had undergone apoptosis (Figure $2 \mathrm{~g}$ ).

Toll-like receptor agonists have been previously shown to delay neutrophil apoptosis. ${ }^{4,27}$ We then analyzed whether they are able to modulate the antiapoptotic effect mediated by flagellin. In these experiments neutrophils were cultured for $24 \mathrm{~h}$ with the TLR1/2 ligand $\mathrm{PAM}_{3} \mathrm{CSK}_{4}$, the TLR2/6 ligand $\mathrm{PAM}_{2} \mathrm{CSK}_{4}$, and the TLR4 ligand LPS, either in the absence or presence of FliC. Apoptosis was then evaluated by fluorescence microscopy. Consistent with previous studies, we found that all these TLR ligands delayed neutrophil apoptosis; however, none of them further increased the antiapoptotic effect exerted by flagellin (Figure 3a). Similar findings were observed when apoptosis was evaluated after $48 \mathrm{~h}$ of culture (data not shown).

Additional studies indicated that FliC was able to not only delay spontaneous apoptosis but also markedly inhibit apoptosis induced by either agonistic antibodies directed to Fas or the phagocytic stimulus Zymosan (Figure 3b). 
a

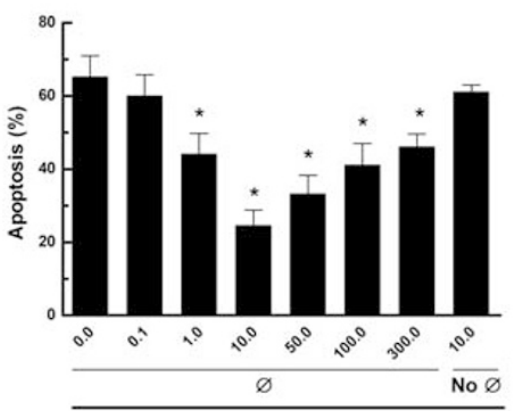

FliC concentration $(\mathrm{ng} / \mathrm{ml})$
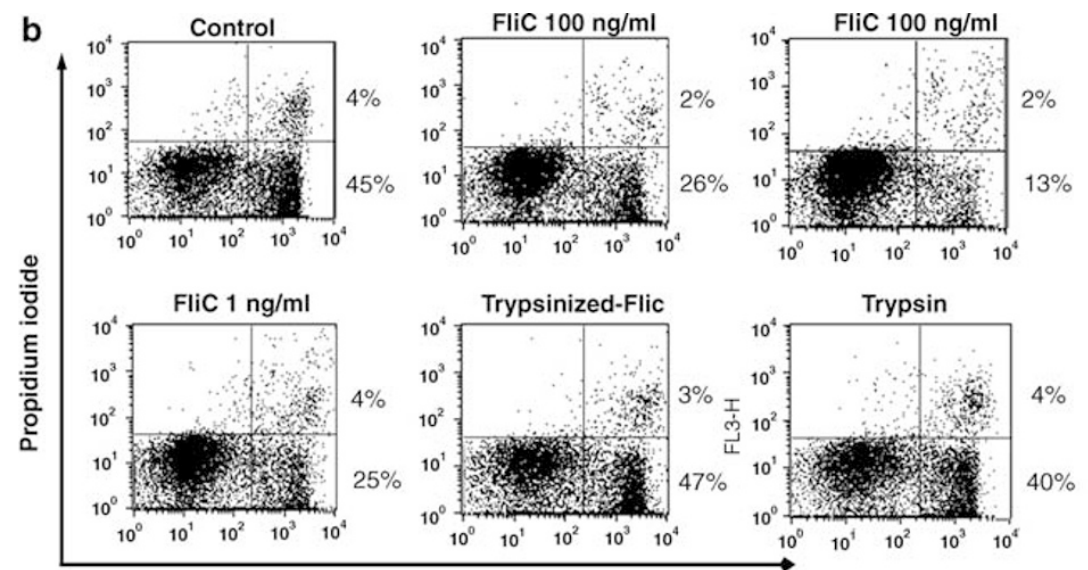

FITC -Annexin V c

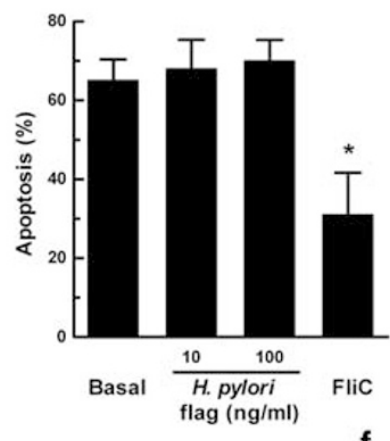

d

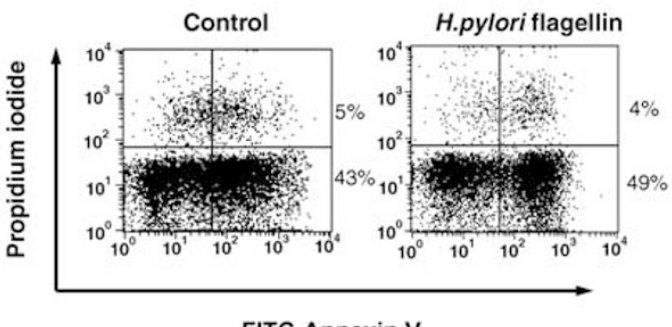

FITC-Annexin V

g

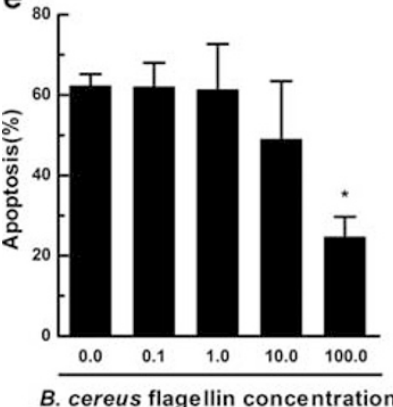

(ng/ml)

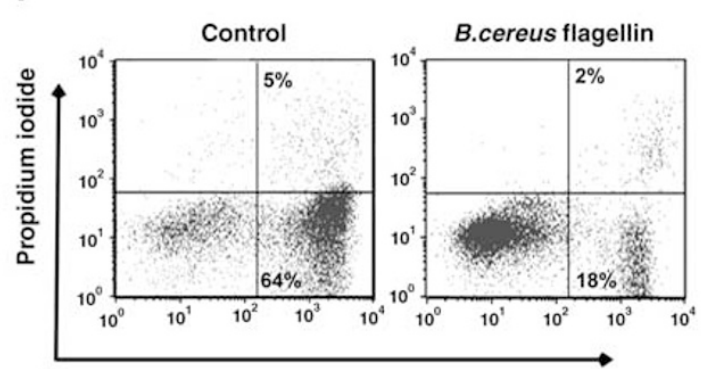

FITC-Annexin V

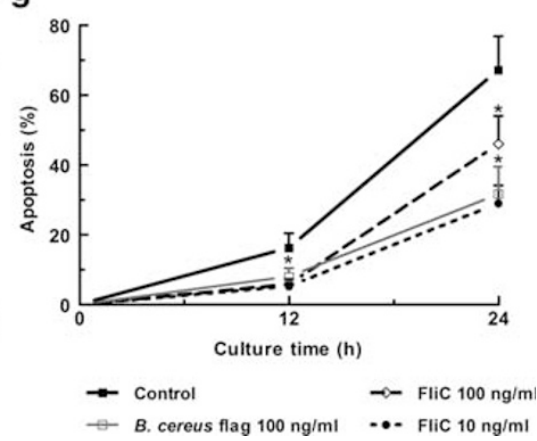

Figure 2 Flagellin delays human neutrophil apoptosis. Neutrophils were cultured with the indicated concentrations of heat-treated flagellin from either S. thyphimurium (FliC), B. cereus, or $\mathrm{H}$. pylori for $24 \mathrm{~h}$ at $37^{\circ} \mathrm{C}$. Apoptosis was then measured (a, c, e) by fluorescence microscopy, ${ }^{\star} P<0.01 \mathrm{vs}$ unstimulated cells, $n=5(\mathbf{a}), n=3(\mathbf{c})$, and $n=4(\mathbf{e})$; or $(\mathbf{b}, \mathbf{d}, \mathbf{f})$ by flow cytometry, data are representative of 5,3 , and 4 experiments performed, respectively. $\varnothing$ symbol represents Heat-treated FliC depicted in figure (a) to differenciate it from unheated FliC (No Ø). (g) Time course of apoptosis modulation by flagellin. Neutrophils were incubated in the absence or presence of FliC $(10-100 \mathrm{ng} / \mathrm{ml})$ or flagellin from B. cereus for 12 and $24 \mathrm{~h}$ at $37^{\circ} \mathrm{C}$. Apoptosis was then measured by fluorescence microscopy ( $n=6,{ }^{\star} P<0.05$ vs unstimulated cells).

The Delay of Neutrophil Apoptosis Induced by Flagellin is Associated with a Reduced Activation of Caspase 3 and a Preserved Expression of $\mathrm{Mcl}-1$

To gain insight in the mechanisms responsible for the delay in neutrophil apoptosis induced by flagellin, we analyzed the expression of both activated caspase- 3 and the antiapoptotic protein $\mathrm{Mcl}-1$. In these experiments, neutrophils were cultured for $24 \mathrm{~h}$ in the absence or presence of flagellin, and the levels of cleaved-caspase 3 and Mcl-1 were evaluated in permeabilized cells by flow cytometry. Results in Figure $4 \mathrm{a}$ and b show that incubation with flagellin resulted in lower levels of activated-caspase 3 and higher levels of Mcl-1, respectively, when compared with control neutrophils. These finding were confirmed by western blot analysis (Figure 4c).

\section{Signaling Pathways that are Activated in Neutrophils by Flagellin}

Modulation of neutrophil survival by PAMPs and cytokines has been shown to involve different signaling pathways. Activation of ERK1/2 MAPK, PI3K/Akt, and NF- $\kappa \mathrm{B}$ usually results in the delay of neutrophil apoptosis, whereas activation of p38 MAPK decreases neutrophil survival. ${ }^{27-31}$ To our knowledge, no previous studies have analyzed the signaling pathways activated in isolated neutrophils by flagellin. Thus, 
to delineate the signaling pathways activated by flagellin, neutrophils were stimulated in the presence or absence of FliC for different times, and then the samples were harvested at indicated time points and subjected to western blotting to assess activation of p38 and ERK MAPKs and Akt phosphorylation. Figure $5 a$ shows that FliC activated all pathways
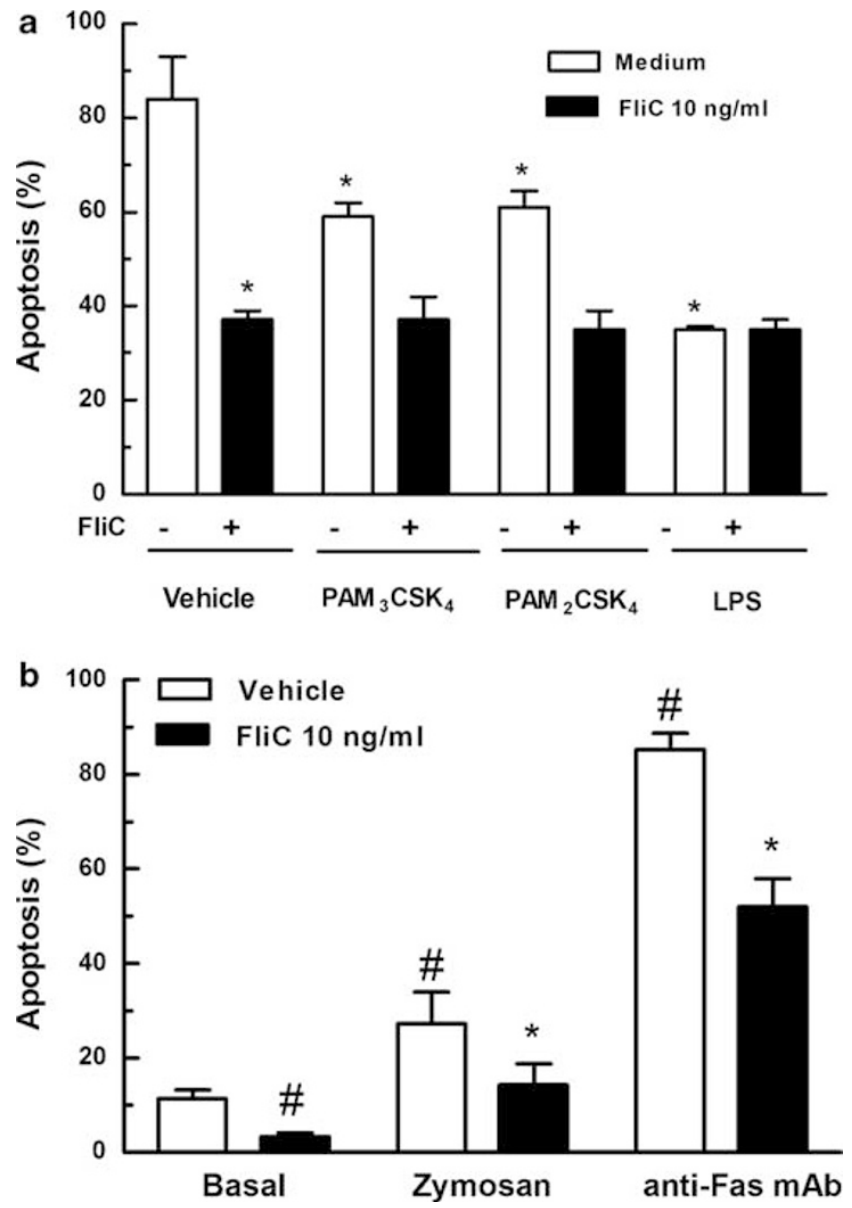

Figure 3 Effect of flagellin on neutrophil survival modulated by different agonists. (a) Neutrophils were cultured with vehicle, $\operatorname{PAM}_{3} \mathrm{CSK}_{4}(1 \mu \mathrm{g} / \mathrm{ml})$, $\mathrm{PAM}_{2} \mathrm{CSK}_{4}(1 \mu \mathrm{g} / \mathrm{ml})$, or LPS $(10 \mathrm{ng} / \mathrm{ml})$ in the presence or absence of FliC $(10 \mathrm{ng} / \mathrm{ml})$ for $24 \mathrm{~h}$ at $37^{\circ} \mathrm{C}$. Apoptosis was then quantified by fluorescence microscopy ( $n=3,{ }^{*} P<0.05$ vs unstimulated cells). (b) Neutrophils were cultured with medium or FliC $(10 \mathrm{ng} / \mathrm{ml})$ for $15 \mathrm{~min}$ at $37^{\circ} \mathrm{C}$ and then for $8 \mathrm{~h}$ at $37^{\circ} \mathrm{C}$ in the presence or absence of Zymosan $(200 \mu \mathrm{g} / \mathrm{ml})$ or a specific agonist $\mathrm{mAb}$ against Fas receptors $(2 \mu \mathrm{g} / \mathrm{ml})$. Apoptosis was then quantified by fluorescence microscopy $(n=3, " \#<0.05$ vs basal without FliC and ${ }^{\star} P<0.05$ vs Zymosan or anti-FAS without FliC).

Figure 4 Inhibition of neutrophil apoptosis by flagellin is associated with a reduced activation of caspase-3 and increased expression of $\mathrm{Mcl}-1$. Neutrophils were cultured in the presence or absence of FliC $(10 \mathrm{ng} / \mathrm{ml})$ for $24 \mathrm{~h}$ at $37^{\circ} \mathrm{C}$. The expression of the activated form of caspase-3 (a) and the expression of the antiapoptotic protein $\mathrm{Mcl}^{-1}$ (b) were then evaluated by flow cytometry $(n=3)$. (c) Western blot analysis of extracts of fresh neutrophils or neutrophils cultured for $24 \mathrm{~h}$ in the absence or presence of $10 \mathrm{ng} / \mathrm{ml}$ of FliC using anti-caspase-3 and Mcl-1-specific mAbs.

Representative experiments are depicted $(n=2)$. a

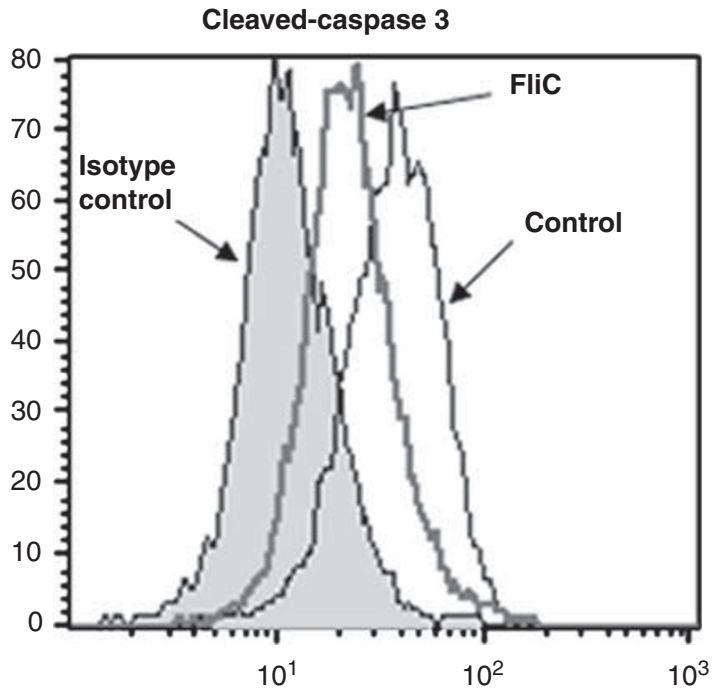

b

$\mathrm{Mcl}-1$

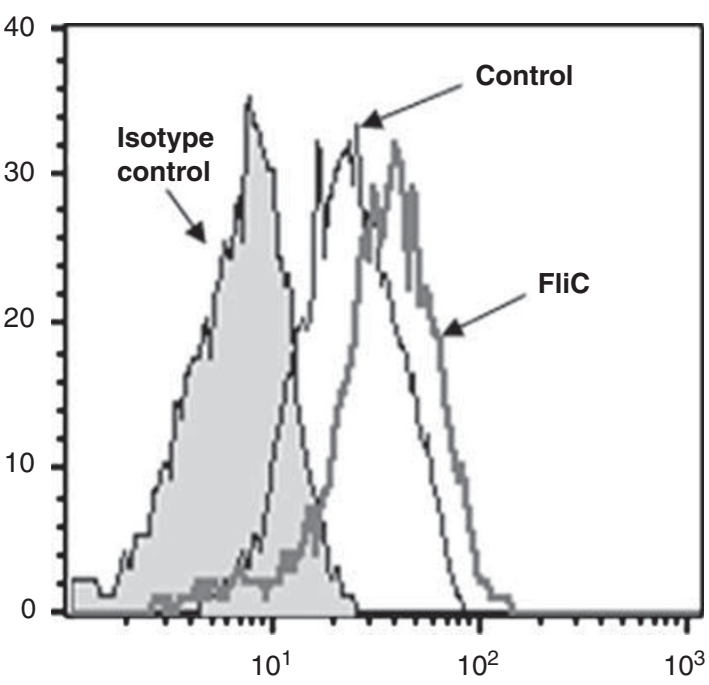

$\begin{array}{llll}\text { C } \text { Time (h) } & 0 & 24 & 24\end{array}$

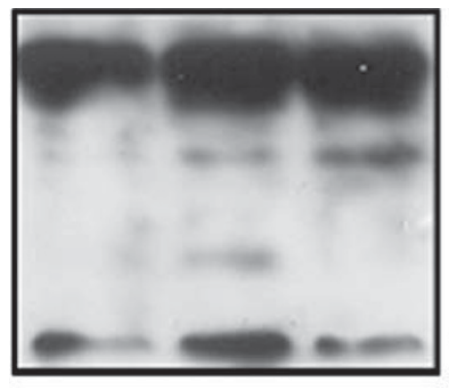

Procaspase-3

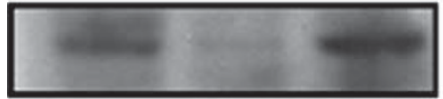

cleaved

Caspase-3

Mcl-1

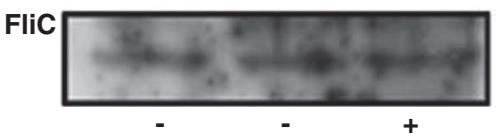

$\beta$-tubulin 


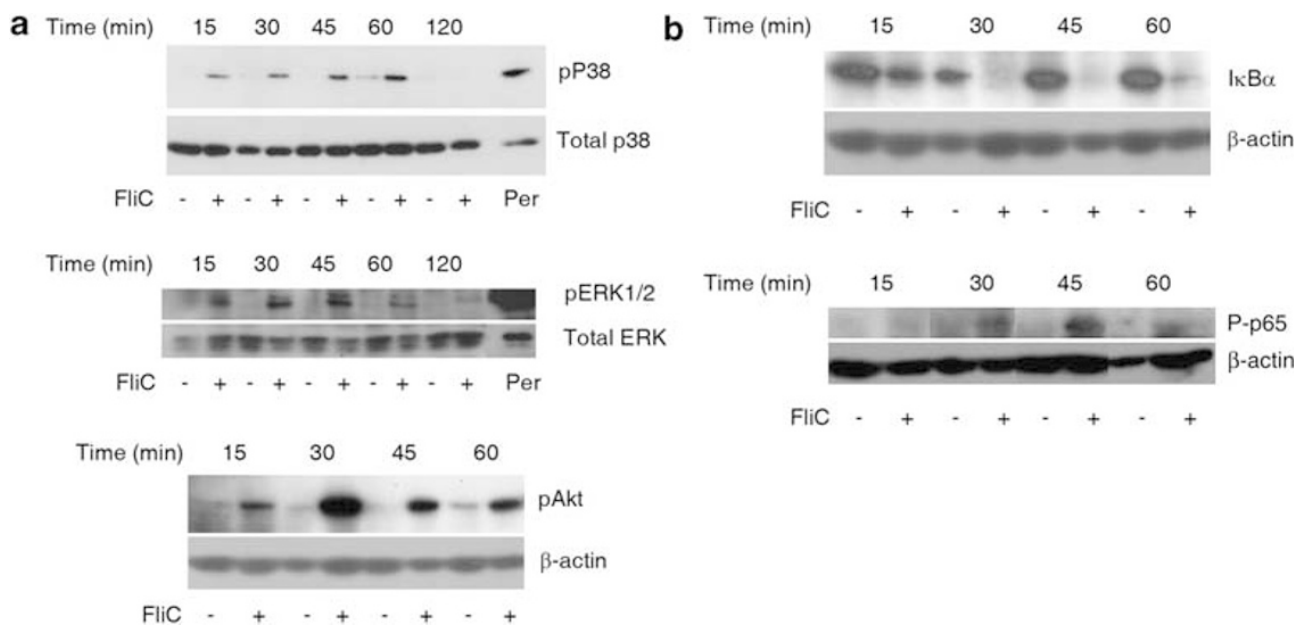

Figure 5 Neutrophil signaling pathways activated by flagellin. (a) Time course of p38, ERK, and Akt phosphorylation triggered by FliC. Neutrophils were incubated in the presence or absence of FliC $50 \mathrm{ng} / \mathrm{ml}$ for the indicated times at $37^{\circ} \mathrm{C}$. Kinase phosphorylation was then assessed by western blot. Immunoblots are representatives of at least three independent experiments. (b) Time course of $1 \kappa \mathrm{B} \alpha$ degradation and p65 phosphorylation induced by flagellin. Neutrophils were incubated in the presence or absence of FliC $50 \mathrm{ng} / \mathrm{ml}$ for the indicated times at $37^{\circ} \mathrm{C}$. Then, I $/ \mathrm{B} \alpha$ degradation and Ser536-p65 phosphorylation were assessed by western blot. Immunoblots are representative of three independent experiments.

analyzed. The phosphorylation of p38 MAPK was observed from 15 to 60 min after stimulation with flagellin, whereas by $120 \mathrm{~min}$ it was not further detected. Similarly, ERK phosphorylation was mainly observed from 15 to $60 \mathrm{~min}$ after FliC stimulation. Phosphorylation of Akt was detected at $15 \mathrm{~min}$ after stimulation. It reached a maximum at $30 \mathrm{~min}$ and remained detectable even at $60 \mathrm{~min}$ after stimulation, which is the last time point determined. We also found that flagellin induced the degradation of $\mathrm{I} \kappa \mathrm{B} \alpha$ (Figure $5 \mathrm{~b}$ ), suggesting its ability to trigger the activation of the NF- $\kappa \mathrm{B}$ pathway. Furthermore, FliC induced Ser536-p65 subunit phosphorylation, a phenomena that might modulate NF- $\kappa \mathrm{B}$ transcriptional activity (Figure 5b).

\section{Involvement of ERK1/2 and NF- $\kappa$ B Pathways in the Delay of Neutrophil Apoptosis Induced by Flagellin}

To determine the signaling pathways responsible for the antiapoptotic effect of flagellin, we used the MEK-ERK kinase inhibitor PD98059, the p38MAPK inhibitor SB203580, the PI3K/Akt inhibitor wortmannin, and the inhibitor of $\mathrm{I} \kappa \mathrm{B} \alpha$ phosphorylation BAY-11-7082. Neutrophils were cultured for $24 \mathrm{~h}$ with or without flagellin, in the absence or presence of these inhibitors, and the levels of neutrophil apoptosis were analyzed by fluorescence microscopy or by using FITC-annexin-V and flow cytometry. None of the inhibitors tested modulated the rate of apoptosis of neutrophils cultured in the absence of flagellin (spontaneous apoptosis; Figure 6a and b). Both PD98059 and BAY-11-7082 partially prevented the antiapoptotic effect induced by flagellin, supporting a role for ERK $1 / 2$ kinase and the NF- $\kappa \mathrm{B}$ pathways in the prevention of neutrophil apoptosis triggered by flagellin.
As the synthesis of many antiapoptotic cytokines is dependent on NF- $\kappa \mathrm{B}$, we performed additional experiments to analyze whether a cytokine released by FliC-stimulated neutrophils could be exerting an autocrine apoptosis-delaying effect. To this end, neutrophils were stimulated with FliC ( $10 \mathrm{ng} / \mathrm{ml}$ ) for $60 \mathrm{~min}$ at $37^{\circ} \mathrm{C}$, and then $\mathrm{FliC}$ was washed out and neutrophils were further incubated for $6 \mathrm{~h}$. Afterward, culture supernatants were recovered and used to test their antiapoptotic properties over fresh neutrophils. Supernatants from cultures of nonstimulated neutrophils were used as controls. Supernatants from FliC-stimulated neutrophils did not modulate neutrophil survival (Figure $6 \mathrm{c}$ ), although the antiapoptotic cytokines IL- $1 \beta$ and IL- 8 were detected by ELISA (data not shown). In line with these findings, we determined that the presence of the IL-1 receptor antagonist did not modify the apoptosis-delaying effect exerted by flagellin (data not shown).

\section{The Presence of Flagella Increases the Ability of Bacteria to Delay Neutrophil Apoptosis}

Bacteria express different PAMPs that are able to delay neutrophil survival. To evaluate the possible contribution of flagellin in the ability of whole bacteria to modulate neutrophil apoptosis, we used wild-type $S$. typhimurium strain (SIN22) and the isogenic aflagellate mutant strain (SIN41). Neutrophils were cultured with heat-killed SIN22 or SIN41 for $24 \mathrm{~h}$ in bacteria/neutrophil ratios ranging from 20:1 to 1:2. Apoptosis was then evaluated using FITC-annexin-V. The results in Figure $7 \mathrm{a}$ and $\mathrm{b}$ show that both bacterial strains markedly inhibited apoptosis. Interestingly, when assessed in low bacteria/neutrophil ratios, flagellate bacteria showed a higher ability than aflagellate bacteria to delay neutrophil 


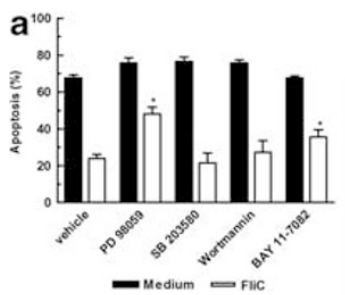

C

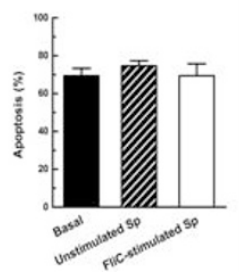

b
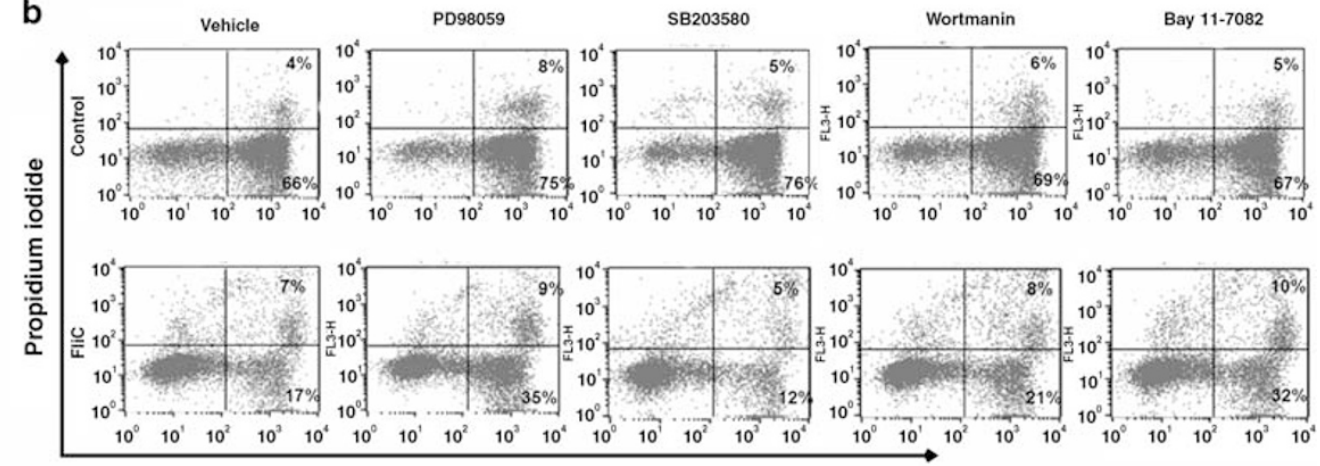

FITC-Annexin V

Figure 6 Effect of pharmacological inhibitors of p38, MEK, PI3K/Akt, or NF- $\kappa$ B pathways on the ability of flagellin to inhibit neutrophil spontaneous apoptosis. Neutrophils were pretreated for $30 \mathrm{~min}$ at $37^{\circ} \mathrm{C}$ with SB203580 (p38 inhibitor; $5 \mu \mathrm{M}$ ), PD98058 (MEK inhibitor; $25 \mu \mathrm{M}$ ), wortmannin (PI3K/Akt inhibitor; $50 \mathrm{nM}$ ), or BAY 11-7082 (IKB $\alpha$ phosphorylation inhibitor; $2.5 \mu \mathrm{M})$. They were then cultured in the presence or absence of FliC $10 \mathrm{ng} / \mathrm{ml}$ for $24 \mathrm{~h}$ at $37^{\circ} \mathrm{C}$. Apoptosis levels were evaluated by fluorescence microscopy (a) or by flow cytometry (b). (a) Results represent mean \pm s.e.m. of five experiments $\left({ }^{*} P<0.01\right.$ vs vehicle). (b) Dot plots are representative of three independent experiments. (c) Neutrophils were incubated for $24 \mathrm{~h}$ at $37{ }^{\circ} \mathrm{C}$ in the absence or presence of 6 -h culture supernatants from $4 \times 10^{6}$ neutrophils/ml, previously stimulated or not with FliC (10 ng/ml). Apoptosis was then evaluated by fluorescence microscopy $(n=4)$.

a
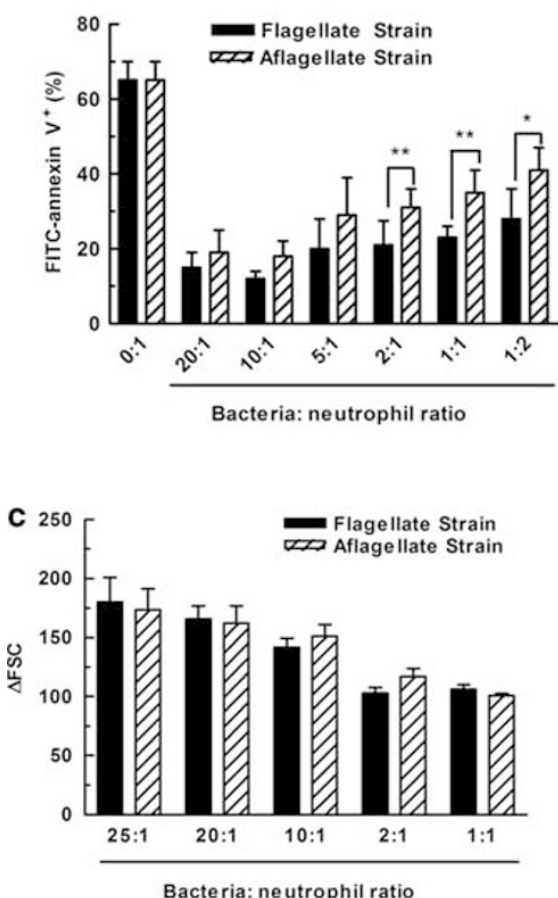

b

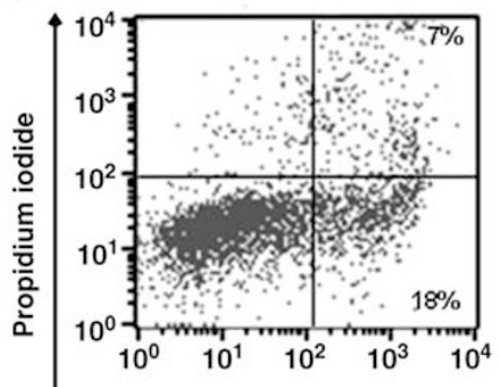

Aflagellate Strain

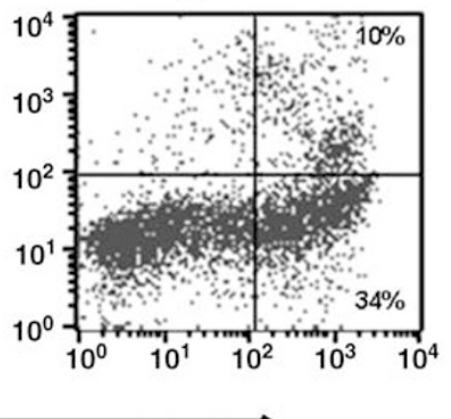

FITC-annexin V
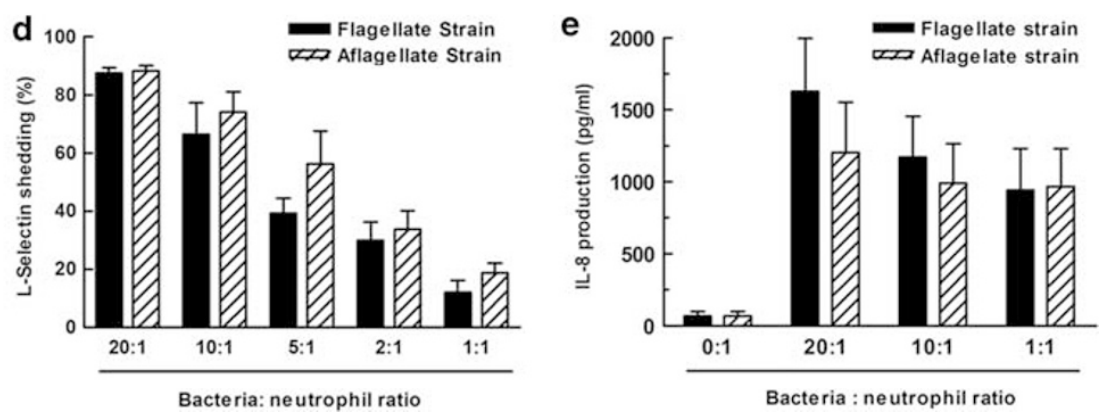

Figure $7(\mathbf{a}, \mathbf{b})$ Modulation of neutrophil apoptosis by flagellate and aflagellate $S$. typhimurim strains. Neutrophils were cultured in the presence or absence of either a heat-killed wild-type S. typhimurium strain (SIN22) or an isogenic aflagellate mutant strain (SIN41) in the indicated bacteria/neutrophil ratios for $24 \mathrm{~h}$ at $37^{\circ} \mathrm{C}$. Apoptosis was then measured by flow cytometry (a; $n=8,{ }^{*} P<0.01$ and ${ }^{*} p<0.05$ vs unstimulated cells). (b) A representative experiment performed in a 1:1 bacteria/neutrophil ratio is shown. (c-e) Activation of neutrophil responses by flagellate and aflagellate $S$. typhimurim strains. Neutrophils were cultured in the presence or absence of either a heat-killed wild-type S. typhimurium strain (SIN22) or an isogenic aflagellate mutant strain (SIN41) in the indicated bacteria/neutrophil ratios for $30 \mathrm{~min}(\mathbf{c}, \mathbf{d})$ or $3 \mathrm{~h}(\mathbf{e})$ at $37^{\circ} \mathrm{C}$. The shape change $(\mathbf{c} ; n=4)$ and the L-selectin expression (d; $n=6$ ) were then evaluated by flow cytometry, whereas the release of IL-8 to culture supernatants (e) was determined by ELISA ( $n=6)$.

apoptosis. Differences in the capacity of both strains to delay neutrophil apoptosis seemed not to be related to differences in their ability to induce neutrophil activation. In fact, both strains showed a similar ability to induce neutrophil activation, as revealed by the evaluation of shape change responses and shedding of L-selectin and IL-8 production (Figure 7c-e). 


\section{DISCUSSION}

In this study we show that flagellin delays neutrophil apoptosis. The concentrations of flagellin $(1-300 \mathrm{ng} / \mathrm{ml})$ that were able to exert antiapoptotic effects were around those found in the plasma of patients with sepsis, in which flagellin levels amounted to $7.1 \pm 0.1 \mathrm{ng} / \mathrm{ml}^{32}$ The apoptosis-delaying effect was independent of the bacterial flagellin source used, as not only S. thyphimurium flagellin but also B. cereus flagellin delayed neutrophil apoptosis. Our findings indicating that H. pylori flagellin, which is not recognized by TLR $5,{ }^{26}$ was unable to exert neutrophil antiapoptotic effects strongly suggest that this receptor is responsible for the delay in neutrophil apoptosis induced by $S$. typhimurium and B. cereus flagellin. The signaling moiety of flagellin is usually hidden in the flagellum but becomes accessible in its monomeric or oligomeric form. The TLR5 detects a specific conformation of flagellin domain D1, which is required for flagellum formation and function, and is not exposed while flagellum remains polymerized. ${ }^{11,33}$ Consequently, flagellin monomers/oligomers are able to induce TLR5 signaling, whereas filamentous flagella do not. ${ }^{33}$ In spite of this, by using two isogenically matched heat-killed wild-type and flagellin-deficient Salmonella, we observed that both strains were able to markedly inhibit neutrophil apoptosis in all the bacteria/neutrophil ratios assessed. It is noteworthy that the flagellate strain exerted a more marked antiapoptotic effect that reached statistical significance in low bacteria/neutrophil ratios $(2: 1$ to $1: 2)$. This could be because of the shedding of flagellin monomers/oligomers during the manipulation of the heat-killed bacteria used in this experiment.

Unexpectedly, the greater capacity to prevent neutrophil

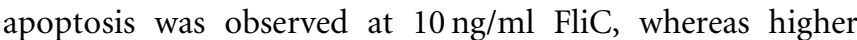
concentrations showed a decreased antiapoptotic effect. A similar biphasic response was previously reported when evaluating TNF- $\alpha$ production induced by the same flagellin concentrations in mouse alveolar macrophages. ${ }^{34}$ As flagellin monomers can polymerize in solution under certain conditions, reconstituting flagellar fibers undistinguishable from native flagella, ${ }^{35}$ it is possible that increasing FliC concentrations tend to more easily re-aggregate, reducing the ability of FliC preparation to prevent neutrophil apoptosis. On the other hand, it has been previously shown that flagellin induces self-tolerance by blocking the release of IRAK from the TLR5 complex in flagellin-tolerant cells. ${ }^{36}$ Consequently, in increasing FliC concentrations, neutrophils might become gradually tolerant to the antiapoptotic effects of flagellin.

Our results seem to be in disagreement with those of Francois et al, ${ }^{27}$ who reported that LPS (TLR4), peptidoglycan (TLR2), R-848 (TLR7/8), and CpG-DNA (TLR9), but not flagellin (TLR5), delay spontaneous apoptosis of neutrophils. In their study, S. typhimurium flagellin used at $100 \mathrm{ng} / \mathrm{ml}$ did not affect neutrophil survival, neither in whole blood samples nor in isolated neutrophils. The reasons for the discrepancies between their results and ours could be because of differences in the experimental settings of both studies. All our experiments were performed using heattreated flagellin whereas they used untreated flagellin. Heating of flagellin leads to the depolymerization of flagellin, thus increasing the signaling capacity of the preparation. ${ }^{11,33}$ Thus, the results of Francois et $\mathrm{al}^{27}$ could merely reflect the incapacity of flagellin polymers to interact with TLR5. A similar reasoning could be applied to explain their inability to detect IKK phosphorylation and Akt activation by flagellin. On the hand, they evaluated neutrophil apoptosis at early time points (at $8 \mathrm{~h}$ of culture), whereas we evaluated apoptosis after 12 and $24 \mathrm{~h}$ of culture.

Studies performed by Hayashi et al ${ }^{19}$ showed that human neutrophils express TLR5 and also that flagellin induces neutrophil activation. However, the transductional pathways activated by flagellin in neutrophils were not characterized. We found that flagellin induces the activation of p38, ERK1/ 2 , and Akt signaling. Moreover, our findings suggest that flagellin also induces NF- $\kappa \mathrm{B}$ activation, as judged by its ability to stimulate $\mathrm{I} \kappa \mathrm{B} \alpha$ degradation and the phosphorylation of its p65 subunit, an event that is known to modulate NF- $\kappa$ B transcriptional activity. By using specific inhibitors, we found that the ERK1/2 MAPK pathway and NF- $\kappa$ B activation seem to be involved in the delay of spontaneous apoptosis induced by flagellin. These observations are in keeping with previous studies showing that activation of ERK MAPK and NF- $\kappa$ B pathways delays neutrophil apoptosis. ${ }^{27-30}$ In fact, the ERK MAPK pathway is involved in the delay of neutrophils apoptosis induced by LPS, bacterial DNA, GM-CSF, and IL-8, ${ }^{28,30-31}$ whereas the involvement of $\mathrm{NF}-\kappa \mathrm{B}$ in the delaying of neutrophil apoptosis induced by TLR2, TLR4, TLR7/8, and TLR9 agonists has also been reported. ${ }^{27,30}$

We also observed that flagellin caused an intense phosphorylation of Akt protein, a PI3K substrate. However, inhibition of the PI3K/Akt pathway did not prevent the delay of apoptosis induced by flagellin. This finding seems to be in contrast with previous studies showing that the PI3K/Akt pathway is involved in the antiapoptotic effect mediated by TLR2, TLR4, TLR7/8, and TLR9 agonists. ${ }^{27-28,31}$ However, it should be noted that the involvement of PI3K in these studies was suggested on the basis of results obtained using high concentrations of wortmannin $(100 \mathrm{nM}-2.5 \mu \mathrm{M})$, which is known to be able to inhibit signaling pathways not related to PI3k/Akt. ${ }^{37}$

Our results indicate that when used together with flagellin, other TLRs agonists that are known to delay neutrophil apoptosis, such as LPS and lipoproteins, ${ }^{27,30}$ were unable to further increase the delaying effect exerted by flagellin on neutrophil apoptosis. We speculate that, as apoptosis prevention is regulated by a set of common transductional pathways activated by different TLR agonists, once a threshold signal is received by the cell, a stereotyped antiapoptotic response is triggered. Moreover, it is well known that activation of TLR results not only in the stimulation of neutrophil function, but also in the induction of inhibitory 
signaling pathways, which involve MyD88s, SOCS1, and IRAK-M. ${ }^{38}$ These inhibitory pathways could limit the functional consequences associated with the activation of multiple TLR.

Interestingly, we also found that flagellin not only inhibited neutrophil spontaneous apoptosis but also that induced by cross-linking of Fas receptors or by the phagocytic stimulus Zymosan. ${ }^{3}$ These findings suggest that under an inflammatory condition, flagellin could be able to modulate neutrophil survival even in the presence of proapoptotic stimuli.

Our findings indicated that only heat-treated flagellin was able to exert an antiapoptotic effect. Previous studies showed that flagellin is secreted into the medium by the flagella secretory apparatus during flagella biosynthesis, in amounts sufficient to cause a proinflammatory immune response. ${ }^{39-40}$ Other reports speculated that monomeric or oligomeric flagellin could be also released by the action of proteolytic enzymes, bile salts, or surfactants present in mucosal sites. ${ }^{41}$ Moreover, evidence for the release of proinflammatory flagellin monomers in vivo was recently reported by Subramanian and $\mathrm{Qadri}^{42}$, who showed that not only the interaction of Salmonella and intestinal epithelial cells promoted monomeric flagellin release but also that this flagellin was not derived from shearing or depolymerization of flagella present on the surface of the bacterium but instead was newly synthesized and secreted after bacterial sensing of host-produced lysophospholipids. Considering that colonization of the human intestine by $S$. typhimurium leads to infiltration of neutrophils into the intestinal epithelium culminating in the formation of an intestinal crypt abscess ${ }^{16}$ it is possible that neutrophils encounter monomeric flagellin under such scenarios. Moreover, in Bowel disease patients' mucosal lesion, neutrophil infiltration represents one of the characteristic features. ${ }^{43}$ In this inflammatory condition, it was proposed that flagellin from commensal bacteria might gain access to the basolateral surface. ${ }^{43}$ Results of our study suggest that under such secenario, flagellin might contribute to perpetuate inflammation by delaying neutrophil apoptosis.

\section{ACKNOWLEDGEMENT}

We thank Mabel Horvat and Beatriz Loria for their valuable assistance. This work was supported by grants from Agencia Nacional de Promoción Científica y Tecnológica (PICT20433), Consejo Nacional de Investigaciones Científicas y Técnicas (PIP6133), Universidad de Buenos Aires, and Fundación Alberto Roemmers (Argentina).

\section{DISCLOSURE/CONFLICT OF INTEREST}

The authors declare no conflict of interest.

1. Kantari C, Pederzoli-Ribeil M, Witko-Sarsat V. The role of neutrophils and monocytes in innate immunity. Contrib Microbiol 2008;15: 118-146.

2. Nathan C. Neutrophils and immunity: challenges and opportunities. Nat Rev Immunol 2006;6:173-182.

3. Maggini J, Raiden S, Salamone G, et al. Regulation of neutrophil apoptosis by cytokines, pathogens and environmental stressors. Front Biosci 2009;14:2372-2385.
4. Colotta F, Re F, Polentarutti N, et al. Modulation of granulocyte survival and programmed cell death by cytokines and bacterial products. Blood 1992;80:2012-2020.

5. Yamamoto C, Yoshida S, Taniguchi H, et al. Lipopolysaccharide and granulocyte colony-stimulating factor delay neutrophil apoptosis and ingestion by guinea pig macrophages. Infect Immun 1993;61: 1972-1979.

6. Liles WC, Dale DC, Klebanoff SJ. Glucocorticoids inhibit apoptosis of human neutrophils. Blood 1995;86:3181-3188.

7. Trevani AS, Andonegui G, Giordano M, et al. Neutrophil apoptosis induced by proteolytic enzymes. Lab Invest 1996;74:711-721.

8. Watson RW, Redmond HP, Wang $\mathrm{JH}$, et al. Neutrophils undergo apoptosis following ingestion of Escherichia coli. J Immunol 1996;156:3986-3992.

9. Gamberale R, Giordano M, Trevani AS, et al. Modulation of human neutrophil apoptosis by immune complexes. J Immunol 1998;161:3666-3674.

10. Steiner TS. How flagellin and Toll-like receptor 5 contribute to enteric infection. Infect Immun 2007;75:545-552.

11. Ramos HC, Rumbo M, Sirard JC. Bacterial flagellins: mediators of pathogenicity and host immune responses in mucosa. Trends Microbiol 2004;12:509-517.

12. Hayashi F, Smith KD, Ozinsky A, et al. The innate immune response to bacterial flagellin is mediated by Toll-like receptor 5 . Nature 2001;410:1099-1103.

13. Smith KD, Ozinsky A. Toll-like receptor-5 and the innate immune response to bacterial flagellin. Curr Top Microbiol Immunol 2002;270:93-108.

14. Miao EA, Andersen-Nissen E, Warren SE, et al. TLR5 and Ipaf: dual sensors of bacterial flagellin in the innate immune system. Semin Immunopathol 2007;29:275-288.

15. McDermott PF, Ciacci-Woolwine F, Snipes JA, et al. High-affinity interaction between Gram-negative flagellin and a cell surface polypeptide results in human monocyte activation. Infect Immun 2000;68:5525-5529.

16. Gewirtz AT, Simon Jr PO, Schmitt CK, et al. Salmonella typhimurium translocates flagellin across intestinal epithelia, inducing a proinflammatory response. J Clin Invest 2001;107:99-109.

17. Sierro F, Dubois $B$, Coste $A$, et al. Flagellin stimulation of intestinal epithelial cells triggers CCL20-mediated migration of dendritic cells. Proc Natl Acad Sci USA 2001;98:13722-13727.

18. Crellin NK, Garcia RV, Hadisfar O, et al. Human CD4+ T cells express TLR5 and its ligand flagellin enhances the suppressive capacity and expression of FOXP3 in CD4+CD25+ T regulatory cells. J Immunol 2005; 175:8051-8059.

19. Hayashi F, Means TK, Luster AD. Toll-like receptors stimulate human neutrophil function. Blood 2003;102:2660-2669.

20. Vijay-Kumar $\mathrm{M}, \mathrm{Wu} \mathrm{H}$, Jones $\mathrm{R}$, et al. Flagellin suppresses epithelial apoptosis and limits disease during enteric infection. Am J Pathol 2006;169:1686-1700.

21. Catalano M, Matteo M, Barbolla RE, et al. Helicobacter pylori vacA genotypes, cagA status and ureA-B polymorphism in isolates recovered from an Argentine population. Diagn Microbiol Infect Dis 2001;41:205-210.

22. Boyum A. Separation of leukocytes from blood and bone marrow. Introduction. Scand J Clin Lab Invest Suppl 1968;97:7.

23. Salamone G, Giordano M, Trevani AS, et al. Promotion of neutrophil apoptosis by TNF-alpha. J Immunol 2001;166:3476-3483.

24. Coxon A, Tang T, Mayadas TN. Cytokine-activated endothelial cells delay neutrophil apoptosis in vitro and in vivo. A role for granulocyte/ macrophage colony-stimulating factor. J Exp Med 1999;190:923-934.

25. Homburg $C H$, de Haas $M$, von dem Borne $A E$, et al. Human neutrophils lose their surface Fc gamma RIII and acquire annexin V binding sites during apoptosis in vitro. Blood 1995;85:532-540.

26. Andersen-Nissen $\mathrm{E}$, Smith KD, Strobe KL, et al. Evasion of Toll-like receptor 5 by flagellated bacteria. Proc Natl Acad Sci USA 2005;102:9247-9252.

27. Francois S, El Benna J, Dang PM, et al. Inhibition of neutrophil apoptosis by TLR agonists in whole blood: involvement of the phosphoinositide 3-kinase/Akt and NF-kappaB signaling pathways, leading to increased levels of Mcl-1, A1, and phosphorylated Bad. $\mathrm{J}$ Immunol 2005;174:3633-3642. 
28. Klein JB, Rane MJ, Scherzer JA, et al. Granulocyte-macrophage colonystimulating factor delays neutrophil constitutive apoptosis through phosphoinositide 3-kinase and extracellular signal-regulated kinase pathways. J Immunol 2000;164:4286-4291.

29. Ward C, Chilvers ER, Lawson MF, et al. NF-kappaB activation is a critical regulator of human granulocyte apoptosis in vitro. J Biol Chem 1999;274:4309-4318.

30. Sabroe I, Prince LR, Jones EC, et al. Selective roles for Toll-like receptor (TLR)2 and TLR4 in the regulation of neutrophil activation and life span. J Immunol 2003;170:5268-5275.

31. Jozsef $L$, Khreiss T, Filep JG. CpG motifs in bacterial DNA delay apoptosis of neutrophil granulocytes. FASEB J 2004;18:1776-1778.

32. Liaudet L, Szabo C, Evgenov OV, et al. Flagellin from Gram-negative bacteria is a potent mediator of acute pulmonary inflammation in sepsis. Shock 2003;19:131-137.

33. Smith KD, Andersen-Nissen E, Hayashi F, et al. Toll-like receptor 5 recognizes a conserved site on flagellin required for protofilament formation and bacterial motility. Nat Immunol 2003:4:1247-1253.

34. Lu W, Hisatsune A, Koga T, et al. Cutting edge: enhanced pulmonary clearance of Pseudomonas aeruginosa by Muc1 knockout mice. J Immunol 2006;176:3890-3894.

35. lino T. Genetics and chemistry of bacterial flagella. Bacteriol Rev 1969:33:454-475.
36. Mizel SB, Snipes JA. Gram-negative flagellin-induced self-tolerance is associated with a block in interleukin-1 receptor-associated kinase release from toll-like receptor 5 . J Biol Chem 2002;277:22414-22420.

37. Capodici C, Hanft S, Feoktistov M, et al. Phosphatidylinositol 3-kinase mediates chemoattractant-stimulated, CD11b/CD18-dependent cellcell adhesion of human neutrophils: evidence for an ERK-independent pathway. J Immunol 1998;160:1901-1909.

38. Liew FY, Xu D, Brint EK, et al. Negative regulation of Toll-like receptormediated immune responses. Nat Rev Immunol 2005;5:446-458.

39. Komoriya K, Shibano N, Higano T, et al. Flagellar proteins and type IIIexported virulence factors are the predominant proteins secreted into the culture media of Salmonella typhimurium. Mol Microbiol 1999;34:767-779.

40. Rochon $\mathrm{M}$, Romling $\mathrm{U}$. Flagellin in combination with curli fimbriae elicits an immune response in the gastrointestinal epithelial cell line HT-29. Microbes Infect 2006;8:2027-2033.

41. Rumbo M, Nempont C, Kraehenbuhl JP, et al. Mucosal interplay among commensal and pathogenic bacteria: lessons from flagellin and Tolllike receptor 5. FEBS Lett 2006;580:2976-2984.

42. Subramanian N, Qadri A. Lysophospholipid sensing triggers secretion of flagellin from pathogenic salmonella. Nat Immunol 2006;7:583-589.

43. Gewirtz AT. TLRs in the Gut. III. Immune responses to flagellin in Crohn's disease: good, bad, or irrelevant? Am J Physiol Gastrointest Liver Physiol 2007;292:G706-G710. 